\title{
Liraglutide Attenuates Preestablished Atherosclerosis in Apolipoprotein E-Deficient Mice via Regulation of Immune Cell Phenotypes and Proinflammatory Mediators $\underline{\underline{S}}$
}

\author{
Robyn Bruen, Seán Curley, Sarina Kajani, Gina Lynch, Marcella E. O’Reilly, \\ Eugène T. Dillon, Eoin P. Brennan, Mary Barry, Stephen Sheehan, (1)Fiona C. McGillicuddy, \\ and (1D) Orina Belton
}

\begin{abstract}
School of Biomolecular and Biomedical Science (R.B., O.B.) and School of Medicine (S.C., S.K., E.P.B., F.C.M.), Diabetes Complications Research Centre, and Mass Spectrometry Resource (E.T.D.), University College Dublin (UCD) Conway Institute, UCD, Belfield, School of Public Health, Physiotherapy and Sports Science, UCD, Belfield (G.L., M.E.O'R.), and Vascular Surgery, St. Vincent's University Hospital (M.B., S.S.), Dublin, Ireland
\end{abstract}

Received April 3, 2019; accepted June 26, 2019

\begin{abstract}
We have shown that the glucagon-like peptide-1 receptor agonist (GLP-1RA) liraglutide (Lir) inhibits development of early atherosclerosis in vivo by modulating immune cell function. We hypothesized that Lir could attenuate pre-established disease by modulating monocyte or macrophage phenotype to induce atheroprotective responses. Human atherosclerotic plaques obtained postendarterectomy and human peripheral blood macrophages were treated ex vivo with Lir. In parallel, apolipoprotein E-deficient $\left(\mathrm{ApoE}^{-1-}\right)$ mice received a high-fat, highcholesterol diet to induce atherosclerosis for 8 weeks, after which $\mathrm{ApoE}^{-1-}$ mice received $300 \mu \mathrm{g} / \mathrm{kg}$ of Lir daily or vehicle control for a further 4 weeks to investigate the attenuation of atherosclerosis. Lir inhibited proinflammatory monocyte chemoattractant protein-1 secretion from human endarterectomy samples and monocyte chemoattractant protein-1, tumor necrosis factor- $\alpha$, and interleukin (IL)- $1 \beta$ secretion from human macrophages after ex vivo treatment. An increase in CD206 mRNA and IL-10 secretion was also detected, which implies resolution of inflammation. Importantly, Lir significantly attenuated pre-established atherosclerosis in $\mathrm{ApoE}^{-/-}$mice in the whole aorta and aortic root. Proteomic analysis of $\mathrm{ApoE}^{-1-}$ bone marrow cells showed that Lir upregulated the proinflammatory
\end{abstract}

cathepsin protein family, which was abolished in differentiated macrophages. In addition, flow cytometry analysis of bone marrow cells induced a shift toward reduced proinflammatory and increased anti-inflammatory macrophages. We concluded that Lir attenuates pre-established atherosclerosis in vivo by altering proinflammatory mediators. This is the first study to describe a mechanism through which Lir attenuates atherosclerosis by increasing bone marrow proinflammatory protein expression, which is lost in differentiated bone marrow-derived macrophages. This study contributes to our understanding of the anti-inflammatory and cardioprotective role of GLP-1RAs.

\section{SIGNIFICANCE STATEMENT}

It is critical to understand the mechanisms through which liraglutide (Lir) mediates a cardioprotective effect as many type 2 diabetic medications increase the risk of myocardial infarction and stroke. We have identified that Lir reduces proinflammatory immune cell populations and mediators from plaque-burdened murine aortas in vivo and augments proresolving bone marrow-derived macrophages in attenuation of atherosclerotic disease, which provides further insight into the atheroprotective effect of Lir.
This work was previously published in Bruen R, Curley S, Kajani S, O'Reilly ME, McGillicuddy FC, and Belton O (2018) Glucagon-like peptide-1 receptor agonist, liraglutide, alters immune populations during regression of atherosclerosis. Diabetologia 61 (Suppl 1):S528-S529, and Bruen R, Curley S, Kajani S, O'Reilly ME, Hogan A, O'Shea D, McGillicuddy FC, and Belton O (2018) Glucagon-like peptide-1 receptor agonist liraglutide impacts immune cell phenotypes in apolipoprotein $\mathrm{E}$ deficient mice during progression and regression of pre-established atherosclerosis. Atherosclerosis 275:e1-e16.

This work was previously submitted in part in a Ph.D. thesis, to University College Dublin by Robyn Bruen (March 2019).

This work was supported by a grant from the European Association for the Study of Diabetes Clinical Diabetes Research Programme in Macrovascular Complications of Diabetes supported by AstraZeneca to O.B., F.C.M., R.B., and S.C. and UCD-Wellcome Institutional Strategic Support Fund, jointly supported University of College Dublin, and the SFI-HRB-Wellcome Biomedical Partnership to O.B.

https://doi.org/10.1124/jpet.119.258343.

[S This article has supplemental material available at jpet.aspetjournals.org.

\section{Introduction}

Atherosclerosis is a chronic progressive disease that is characterized by the accumulation and deposition of lipids and fibrous elements coupled with an inflammatory response resulting in the development of an arterial atherosclerotic lesion (Ross, 1993). The earliest clinical hallmark of a developing lesion is the accumulation of lipid-laden macrophages known as foam cells, which aggregate to form the "fatty streak" (Gerhard and Duell, 1999). Fatty streaks are precursors for advanced lesions, where clinical events stem from lesion rupture or erosion and acute occlusions from thrombus formation, which clinically manifest as a myocardial infraction or stroke (Lusis, 2000).

In addition to established risk factors, such as hyperlipidemia and hypertension, it is now accepted that diabetes 
mellitus-associated hyperglycemia and oxidative stress contribute to accelerated-atherosclerosis by modulating monocyte and macrophage function (Bornfeldt and Tabas, 2011). Work over the past decade has identified the importance of monocyte and macrophage cells in atherosclerotic plaque formation. In a simplified model, macrophages are classified as proinflammatory (M1) or anti-inflammatory (M2). M1 macrophages are induced by inflammatory cytokines and sustain the ongoing inflammatory response via generation of tumor necrosis factor- $\alpha$ (TNF- $\alpha$ ). M2 macrophages are induced by interleukin (IL)-10 and IL-4/IL-13 and promote tissue repair and healing (Fujisaka et al., 2009). M1 and M2 macrophages show plasticity in response to stimuli from the microenvironment and have functional roles in other disorders of chronic low-grade inflammation such as obesity and atherosclerosis (Fujisaka et al., 2009). We have previously shown there is an M2-to-M1 switch during atherosclerotic plaque progression in human patients (de Gaetano et al., 2016), and it is now known that type 2 diabetes mellitus is characterized by a reduction in M2 populations, which is supported by in vivo studies, showing that a shift in M1:M2 ratio directly correlates to the development of insulin resistance (Ye et al., 2016). Stabilizing the progression of cardiovascular disease (CVD) remains a major target in people with type 2 diabetes mellitus. Indeed, recently, there has been increased emphasis on identifying the CVD safety and benefit of recently developed glucose-lowering agents.

Increasing evidence suggests that glucagon-like peptide-1 (GLP-1) therapies, including liraglutide (Lir), a GLP-1 receptor agonist (GLP-1RA), may simultaneously impact the pathogenesis of both type 2 diabetes mellitus and CVD (Arakawa et al., 2010; Gaspari et al., 2011; Nagashima et al., 2011; Rizzo et al., 2015). Dipeptidyl peptidase-4 inhibitors and GLP-1RAs inhibit monocyte and macrophage inflammatory responses and accumulation resulting in the attenuation of atherosclerotic lesion progression in apolipoprotein E knockout $\left(\mathrm{ApoE}^{-1-}\right)$ mice (Arakawa et al., 2010; Nagashima et al., 2011) and improve endothelial cell function and reduce adhesion marker expression (Gaspari et al., 2011). Lir also modulates inflammation and reduces oxidative stress in diabetic patients (Rizzo et al., 2015). In addition, GLP-1RA decrease proinflammatory macrophages in obese type 2 diabetic patients (Hogan et al., 2014) and promote M2 polarization in human monocytes from healthy volunteers (Shiraishi et al., 2012).

We have previously reported that regression of pre-established atherosclerosis in vivo is associated with increased M2 macrophage polarization and IL-10 secretion in the murine aorta (McCarthy et al., 2013). Recently, we have shown Lir can limit the development of early atherosclerosis in $\mathrm{ApoE}^{-1-}$ mice by promoting a proresolving macrophage phenotype (Bruen et al., 2017), which implies that Lir may protect against macrovascular complications of diabetes. To date, a limited number of studies have examined the effect of Lir on immune cell phenotypes in the context of regression of preestablished atherosclerosis. This is important because most patients have established disease at diagnosis of atherosclerosis, with evident cellular and clinical plaques, and the therapeutic goal is to attenuate progression or induce resolution of the disease. This present study is the first to show that Lir stabilizes plaque progression and may induce regression of pre-established atherosclerosis in vivo. In addition, through phenotypic and proteomic analysis of immune cell populations, it provides mechanistic insight into the atheroprotective effect of the GLP-1RA (i.e., Lir) in established atherosclerotic disease.

\section{Materials and Methods}

\section{Human Studies}

Studies were approved by St. Vincent's University Hospital, Dublin Ethics Committee, and adhered to international guidelines and the Declaration of Helsinki principles as revised in 2008. All participants provided informed written consent. Human endarterectomy samples were obtained from consenting patients of mixed sexes postrevascularization surgery and sectioned into relatively disease-free (RDF) and diseased plaque (DP) portions. Plaque portions were cultured in RPMI medium, supplemented with $10 \%$ fetal bovine serum, $1 \%$ L-glutamine, and $100 \mathrm{U}$ of penicillin-streptomycin (P-S) (Bio-sciences, Dublin, Ireland) as described previously (Erbel et al., 2014; Brennan et al., 2018). Plaque sections were treated ex vivo with $1 \mu \mathrm{M}$ Lir (Novo Nordisk, Dublin, Ireland) or phosphate-buffered saline (PBS) (Biosciences) as a vehicle control (VC) for 28 hours. Peripheral blood mononuclear cells (PBMCs) were isolated from whole blood by layering diluted blood in PBS (1:1) onto lymphoprep and centrifuging for 25 minutes at $400 \mathrm{~g}$. The PBMC layer was removed and centrifuged for 5 minutes at $800 \mathrm{~g}$ to remove platelets. Cells were resuspended in M199 medium (Analab, Dublin, Ireland), supplemented with $10 \%$ human serum (Sigma-Aldrich, Wicklow, Ireland) and $100 \mathrm{U}$ of P-S and plated on 10-cm Petri dishes for 2 hours. Adherent cells after 2 hours were considered monocytes, and these cells were counted and reseeded in 12-well plates at a density of $1 \times 10^{6}$ cells/ml M199 medium containing $100 \mathrm{ng} / \mathrm{ml}$ of monocyte-colony stimulating factor (Biolegend, London, UK). Cells were treated with $250 \mathrm{nM}$ Lir or PBS VC for 6 hours in the presence or absence of $100 \mathrm{ng} / \mathrm{ml}$ of lipopolysaccharide (LPS) (InvivoGen, Ireland) for 4 hours and 5 mM ATP (SigmaAldrich) for the final hour of treatments for inflammasome activation. Enzyme-linked immunosorbent assay (ELISA) was carried out on plaque and cell supernatants, and quantitative real-time polymerase chain reaction (qRT-PCR) was performed on differentiated PBMCs.

\section{Animals}

The Animal Research and Ethics Committee, University College Dublin, and the Health Products Regulatory Agency of Ireland approved all protocols; these protocols adhered to institutional and international guidelines. Eight-week-old C57BL/6J ApoE ${ }^{-/-}$male mice (C57BL/6J-ApoE ${ }^{\text {tm1Unc }}, 002052$; RRID:IMSR_TAC:apoe; Charles River, Margate, UK) were housed in specific pathogen-free conditions in 12-hour light/dark cycles. ApoE ${ }^{-1-}$ mice were randomized to receive a high-fat (60\% kcal from fat), high-cholesterol (1\%) diet (HFHCD) (Research Diets Inc, New Brunswick, NJ) for 8 weeks ( $n=10$ per group)

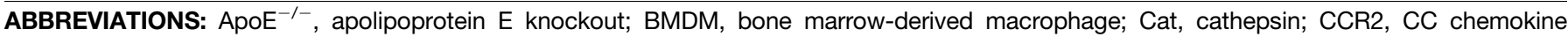

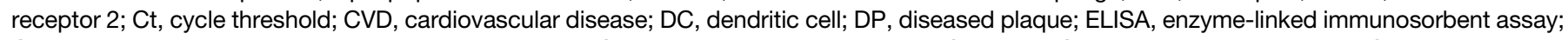

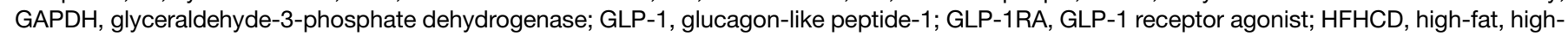

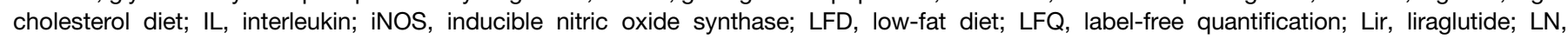

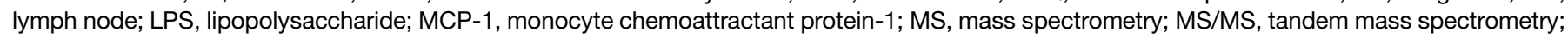

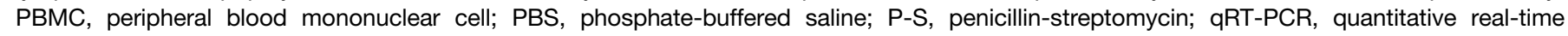
polymerase chain reaction; RDF, relatively disease free; TNF- $\alpha$, tumor necrosis factor- $\alpha$; VC, vehicle control.
} 
to establish disease. For analysis of established disease, 8-week-old $\mathrm{ApoE}^{-/-}$mice were fed a low-fat $(10 \% \mathrm{kcal}$ from fat), $0 \%$ cholesterol diet (LFD) or HFHCD for a total of 12 weeks, in which mice were randomized to receive the HFHCD and received once daily Lir subcutaneous injections from weeks 9 to 12 , titrated upward for 10 days $(1,3,10,30,50,100,150,200,250$, and $300 \mu \mathrm{g} / \mathrm{kg})$ and maintained at $300 \mu \mathrm{g} / \mathrm{kg}(n=21$ per group) or VC PBS from weeks 9 to 12. Mice fed LFD received daily subcutaneous PBS injections from weeks 9 to 12 as a control. Mice were scored daily and euthanized under isoflurane (Duggan Veterinary, Tipperary, Ireland) by a terminal retro-orbital bleed and cervical dislocation. Aortae, lumbar, and inguinal lymph nodes (LNs), spleen, and bone marrow were harvested. The unit of analysis was a single animal or organ. Aortae were analyzed by en face staining ( $n=8$ per group) or cultured ex vivo for 6 hours with aortic tissue analyzed by qRT-PCR ( $n=5-7$ per group), and supernatants were analyzed by ELISA ( $n=7$ per group). Hearts were sectioned for aortic root analysis of atherosclerotic lesions by Oil Red O staining and for $\mathrm{F} 4 / 80^{+}$macrophage cells $(n=3-5$ per group) as detailed in the Supplemental Methods. LNs, spleen, and bone marrow cells were analyzed by flow cytometry $(n=5$ per group), and bone marrow cells were also analyzed by mass spectrometry ( $n=3$ per group).

\section{En Face Whole Aorta Sudan IV and Aortic Root Oil Red O Staining}

En face staining was carried out on whole $\mathrm{ApoE}^{-/-}$aortae, perfused with PBS in vivo, fixed in $10 \%$ neutral buffered formalin, and stained using Sudan IV as in Bruen et al. (2017). ApoE ${ }^{-/-}$hearts were perfused with PBS before freezing in OCT medium over dry ice and were stored at $-80^{\circ} \mathrm{C}$ until cryosectioned at $-25^{\circ} \mathrm{C}$. When the aortic valves were clearly visible, eight $8 \mu \mathrm{m}$ sections per mouse were collected onto Superfrost slides spanning a region of $\sim 800 \mu \mathrm{m}$. Cyrosections were dried at room temperature and frozen at $-80^{\circ} \mathrm{C}$. For Oil Red O aortic root staining, frozen slides were thawed at $37^{\circ} \mathrm{C}$ for 1 minute, air-dried for 1 hour at room temperature, and fixed with $4 \%$ paraformaldehyde for 5 minutes, followed by $60 \%$ isopropanol for 5 minutes. Sections were stained with Oil Red O working solution ( $0.5 \mathrm{~g}$ Oil Red $\mathrm{O}$ in $100 \mathrm{ml}$ of isopropanol, diluted using three parts stock with two parts deionized water, made 24 hours before staining, and $0.22 \mu \mathrm{m}$-filtered) for 10 minutes in the dark. Slides were rinsed three times for 2 seconds each with $60 \%$ isopropanol, followed by a wash with $\mathrm{dH}_{2} \mathrm{O}$. Sections were stained with hematoxylin for 6 minutes, followed by a thorough wash for 10 minutes using $\mathrm{dH}_{2} \mathrm{O}$. Slides were not dried before the addition of two to three drops aqueous mounting medium per slide, followed by a coverslip. Coverslips were allowed to adhere firmly for 30-60 minutes before imaging on a ScopePad550 5 Megpixel Android microscope camera at $4 \times$ magnification.

\section{Bone Marrow-Derived Macrophages}

$\mathrm{ApoE}^{-/-}$bone marrow-derived macrophages (BMDMs) were cultured as previously described (Bruen et al., 2017). Briefly, bone marrow was flushed from femurs and tibiae for proteomics or cultured in 25\% L929-conditioned medium for 7 days. Suspension cells on day 7 of culture represented monocytes, and adherent cells represented macrophages. Both monocytes and BMDMs were analyzed by flow cytometry and BMDMs by qRT-PCR.

\section{Proteomics}

Mass spectrometry analysis was carried out on bone marrow cells taken directly from femurs and tibae from $\mathrm{ApoE}^{-1-}$ mice. Bone marrow cells were lysed in $6 \mathrm{M}$ urea (Sigma-Aldrich), and the protein concentration was determined using a Bradford protein assay (BioRad, Fannin Ltd, Dublin, Ireland) with $40 \mu \mathrm{g}$ of protein stored in $40 \mu \mathrm{l}$ of $6 \mathrm{M}$ urea at $-20^{\circ} \mathrm{C}$. Proteins were trypsin digested with peptides, acidified, and washed.
For trypsin digestion, dithiothreitol (Fisher Scientific, Dublin, Ireland) was used to reduce disulphide bonds of plasma protein samples, followed by 30 minutes of incubation at $60^{\circ} \mathrm{C}$ on a thermomixer without shaking. Samples were briefly centrifuged, and $200 \mathrm{mM}$ iodoacetemide (Sigma-Aldrich) was added to alkylate the samples. dithiothreitol, iodoacetemide, and urea concentrations were diluted using $50 \mathrm{mM}$ ammonium bicarbonate (Sigma-Aldrich) to ensure that $6 \mathrm{M}$ of urea was diluted to $2 \mathrm{M}$ urea before the addition of trypsin, and $0.5 \mu \mathrm{g} / \mu \mathrm{l}$ of trypsin (Sigma-Aldrich) was added to each sample with a working ratio of protein to trypsin 50:1. Digestion was carried out overnight at $37^{\circ} \mathrm{C}$ on a thermomixer set to $350 \mathrm{rpm}$. To stop digestion, acetic acid (1\% final volume) (Fisher Scientific) was added. Peptides were washed twice with $1 \%$ trifluoroacetic acid (Fisher Scientific) in deionized water using a StageTip and centrifuged at 10,000 rpm for 5 minutes. Peptides were acidified using $50 \%$ acetonitrile and $0.1 \%$ trifluoroacetic acid and then centrifuged at $10,000 \mathrm{rpm}$ for 5 minutes and repeated twice. Samples were eluted using $50 \%$ acetonitrile (Fisher Scientific) and $0.1 \%$ trifluoroacetic acid through a StageTip. Evaporation of samples was carried out for 20-30 minutes at $60^{\circ} \mathrm{C}$ using a CentriVap Concentrator. Samples were resuspended in $2.5 \%$ acetonitrile and $0.5 \%$ acetic acid. Protein concentration and confirmation of peptide bonds was evaluated using the NanoDrop2000 at $218 \mathrm{~nm}$. Samples were centrifuged at $13,000 \mathrm{rpm}$ for 5 minutes and transferred to a mass spectrometry vial.

Mass Spectrometry. Peptide samples were analyzed on a quadrupole Orbitrap (Q-Exactive; Thermo Scientific, Dublin, Ireland) mass spectrometer. The Orbitrap was equipped with a reversed-phase NanoLC UltiMate 3000 high-performance liquid chromatograpy system (Dionex LC Packings, now Thermo Scientific). Peptide fractions were loaded onto $\mathrm{C} 18$ reversed-phase columns $(5 \mathrm{~cm}$ length, $75 \mu \mathrm{m}$ inner diameter) and eluted with a linear gradient from $8 \%$ to $40 \%$ acetonitrile with $0.5 \%$ trifluoroacetic acid in 60 minutes at a flow rate of $3 \mu \mathrm{l} / \mathrm{min}$. Five microliters was used as the injection volume. The Orbitrap was operated in a data-dependent mode, automatically switching between mass spectrometry (MS) and MS2 acquisition. Survey full-scan MS spectra $(\mathrm{m} / z 350-1600)$ were with a resolution of 70,000, MS2 spectra with a resolution of 17,500 . The 12 most intense ions were sequentially isolated and fragmented by higher-energy C-trap dissociation (O’Reilly et al., 2016).

Protein Identification. Raw data from the Orbitrap were processed using MaxQuant version 1.5.6.0 (Max Plank Institute of Biochemistry, Munich, Germany). To identify peptides and proteins, tandem mass spectrometry (MS/MS) spectra were matched to the UniProt mouse database. Tryptic specificity allowing two missed cleavages was used for all searches. The database searches were performed with carbamidomethyl as fixed modification and acetylation (protein $\mathrm{N}$ terminus) and oxidation (M) as variable modifications. Mass spectra were searched using the default setting of MaxQuant 1.5.6.0, namely, a false discovery rate of $1 \%$ on the peptide and protein level. Label-free quantitative (LFQ) ion intensities for protein profiles were generated using signals of corresponding peptides in different nano-high-performance liquid chromatograpy. MS/MS runs were matched by MaxQuant 1.5.6.0 applying a mass accuracy of at least $20 \mathrm{ppm}$ and a maximum time window of 1 minute.

Proteomic Data Analysis. MaxQuant 1.5.6.0 identified proteins and generated LFQ intensities, which were analyzed using Perseus version 1.4.1.3 (Max Planck Institute of Biochemistry). Protein identifications were filtered to eliminate the reverse database and common contaminants. Data were filtered with stringent inclusion to obtain peptides in which the peptide was present in all biologic replicates in all samples. Data were log-transformed, and unpaired $t$ test comparisons of fractions were carried out. For heat-map visualization, missing values were imputed with values from the normal distribution. The data set was normalized by $z$-score. Proteins were analyzed for pathways using Ingenuity Pathway Analysis (2018 Qiagen, Manchester, UK). 


\section{Flow Cytometry Analysis}

Day 7 BMDMs, LNs, and spleens, which were homogenized and flushed with PBS, were stained with antibodies described in Table 1. All flow cytometry antibodies were purchased from BD Biosciences (Oxford, UK) (Supplemental Table 1). Flow cytometry controls included single stains consisting of a single antibody mixed with BMDMs, LN, or splenic tissue. For gating strategies, fluorescence minus one control was used, where all antibodies excluding one were incubated with BMDMs, LN, or splenic tissue. Samples were run on the Beckman Coulter CyanADP flow cytometer (Beckman Coulter, Brea, CA) and analyzed using FlowLogic software (Miltenyi Biotec Ltd, Surrey, UK).

\section{ELISA}

ELISA was performed for human TNF- $\alpha$, monocyte chmeoattractant protein-1 (MCP-1), and IL-1 $\beta$ (Thermo Fisher, Dublin, Ireland) and murine TNF- $\alpha$ (R\&D systems, Oxon, UK) according to the manufacturer's instructions.

\section{qRT-PCR}

Cellular RNA was extracted from PBMC-derived human macrophages and $\mathrm{ApoE}^{-/-}$BMDMs using RNeasy Qiagen kits (Qiagen Ltd) per the manufacturer's instructions. Murine aortas were homogenized using the Qiagen Tissue Lyser II and steel beads (Qiagen Ltd) for 10 minutes at $30 \mathrm{~Hz}$, twice before RNA extraction, and extracted using Trizol reagent (Bio-Sciences). Briefly, samples were incubated on ice (5 minutes) with the addition of 1:1 chloroform:isoamyl alcohol (Sigma-Aldrich), vortexed, incubated on ice (8 minute), centrifuged $\left(18,500 \mathrm{~g}, 15\right.$ minutes, $\left.4^{\circ} \mathrm{C}\right)$ with the aqueous layer taken, mixed with cold isopropanol (Fisher Scientific), and stored overnight $\left(-20^{\circ} \mathrm{C}\right)$. Samples were centrifuged $(18,500 g, 30$ minutes) with the RNA pellet washed twice in $75 \%$ cold ethanol (Sigma-Aldrich), resuspended in RNase-free water (Qiagen Ltd.), and quantified using the NanoDrop2000; 100-1000 ng RNA was reverse-transcribed to cDNA and analyzed on a Thermo Fisher Quantstudio7 qRT-PCR machine. SYBR Green human TNF- $\alpha$ (NC_000006), murine TNF- $\alpha$ (Y00467.1), and murine inducible nitric oxide synthase (iNOS) (NC_000017) (Table 2) (Eurofins BPT Ireland Ltd, Waterford, Ireland) sequences are available in GenBank with associated accession numbers, murine Taqman Arg-1 (Mm00475988_m1), cathepsin (Cat)B (Mm01310506_m1) and CatZ (Mm00517697_m1) were analyzed using glyceraldehyde-3phosphate dehydrogenase (GAPDH) (human-Hs02786624_g1, murineMm99999915_g1), and 18S rRNA (Hs99999901_s1) (Bio-sciences) as reference genes. Cycle threshold (Ct) values were analyzed by the $\Delta \Delta \mathrm{Ct}$ method.

\section{TABLE 1}

Flow cytometry antibodies Antibodies used for flow cytometry analysis of bone marrow cells, spleens and lymph nodes (LNs).

\begin{tabular}{|c|c|}
\hline Cell & Antibodies \\
\hline $\begin{array}{l}\text { Inflammatory bone marrow } \\
\text { monocytes }\end{array}$ & $\begin{array}{l}\mathrm{CD} 45^{+} \mathrm{CD}_{115^{+}} \mathrm{F} 4 / 80^{+} \mathrm{Ly} 6 \mathrm{C}^{+} \\
\mathrm{CD}^{-}\end{array}$ \\
\hline Resident bone marrow monocytes & $\begin{array}{l}\mathrm{CD}_{\mathrm{CD}}{ }^{+} \mathrm{CD}_{11} \mathrm{c}^{+} \\
\end{array}$ \\
\hline $\begin{array}{l}\text { M1-like bone marrow-derived } \\
\text { macrophages (BMDMs) }\end{array}$ & $\begin{array}{l}\mathrm{CD}^{2} 5^{+} \mathrm{CD}_{115^{+}} \mathrm{CD}_{11} \mathrm{~b}^{+} \mathrm{F} 4 / \\
80^{+} \mathrm{Ly}^{\mathrm{hi}}\end{array}$ \\
\hline M2-like BMDMs & $\begin{array}{l}\mathrm{CD}_{45^{+}} \mathrm{CD}^{\mathrm{C}} 115^{+} \mathrm{CD}^{+} \mathrm{Cy}^{+} 6 \mathrm{C}^{\mathrm{lo}} \\
\text { F4/ }\end{array}$ \\
\hline $\begin{array}{l}\text { Monocytes and dendritic cells } \\
\text { (DCs) in LNs }\end{array}$ & 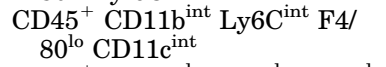 \\
\hline DCs in LNs & $\begin{array}{l}\mathrm{CD}^{2} 5^{+} \mathrm{CD} 11 \mathrm{~b}^{\text {lo }} \text { Ly6C } \mathrm{C}^{\mathrm{lo}} \mathrm{F} 4 / 80^{\text {lo }} \\
\end{array}$ \\
\hline Splenic M1 macrophages & $\begin{array}{l}\mathrm{CD} 45^{+} \text {CD11b }{ }^{\mathrm{hi}} \mathrm{F} 4 / 80^{\mathrm{hi}} \\
\text { CD11 }^{\mathrm{lo}} \mathrm{Ly}^{\mathrm{h}} \mathrm{C}^{\mathrm{hi}}\end{array}$ \\
\hline Splenic M2 macrophages & 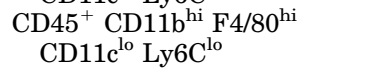 \\
\hline
\end{tabular}

TABLE 2

SYBR green primer sequences

SYBR Green Primer Gene Sequence

Human TNF- $\alpha$ forward 5 '-CTCGAACCCCGAGTGACAA-3

Human TNF- $\alpha$ reverse 5'-GCTGCCCCTCAGCTTGAG-3'

Murine iNOS forward $\quad 5^{\prime}$-CCCTCCTGATCTTGTGTTGGA-3'

Murine iNOS reverse 5'-CCACCCGAGCTCCTGGAAC-3'

Murine TNF- $\alpha$ forward 5'-GGCAGGTCTACTTTGGAGTCATTGC-3'

Murine TNF- $\alpha$ reverse 5'-ACATTCGAGGCTCCAGTGAATTCGG-3'

iNOS, inducible nitric oxide synthase; TNF, tumor necrosis factor.

\section{Statistical Analysis}

All data were analyzed using GraphPad Prism 5.0c (GraphPad Software Inc, San Diego, CA) and are expressed as the mean \pm S.E.M. Shapiro-Wilk normality tests were carried out to determine whether the data were parametric or nonparametric. For comparison of human endarterectomy, DP PBS versus DP Lir and PBMC-derived macrophages VC + LPS versus Lir + LPS paired $t$ tests or Wilcoxonmatched pairs signed rank tests were used to analyze parametric or nonparametric data, respectively. For comparison of LFD versus HFHCD and HFHCD versus LFD, Mann-Whitney tests were used. For multiple comparisons of animal weight, a two-way analysis of variance with Bonferroni's post-test was used. Statistical significance comparing DP PBS versus DP Lir, VC + LPS versus Lir + LPS and LFD versus HFHCD was considered when $* P<0.05$, $* * P<0.01$, and $* * * P<0.001$, and $P>0.05$ was NS and similarly considered when HFHCD versus HFHCD + Lir with ${ }^{\$} P<0.05,{ }^{\$} P P<0.01$, and $\$ \$ \$ P<0.001$.

\section{Results}

Lir Alters Inflammatory Chemokine Secretion from Human Atherosclerotic Plaque. Inflammatory chemokine secretion of MCP-1 from human atherosclerotic plaques cultured postendarterectomy and treated ex vivo with $1 \mu \mathrm{M}$ Lir was analyzed by ELISA. Plaques were sectioned into DP and RDF portions, which served as intraindividual controls. Ex vivo Lir treatment significantly decreased MCP-1 secretion from human DP atherosclerotic plaques (DP VC $79.32 \pm 48.53 \mathrm{ng} / \mathrm{ml}$ vs. DP Lir $58.00 \pm 35.31 \mathrm{ng} / \mathrm{ml}, n=11$, $P<0.01$, whereas MCP-1 from RDF plaques was not altered compared with VC affter Lir treatment (Fig. 1A).

Lir Modulates Monocyte and Macrophage Phenotype. To asses whether Lir can modulate monocyte or macrophage phenotype, we next analyzed proinflammatory cytokines in human PBMC-derived macrophages. Initially, MCP-1 secretion was analyzed similarly to the human endarterectomy samples, where a significant reduction in MCP-1 secretion from human PBMC-derived macrophages treated with Lir + LPS compared with the VC+LPS was detected (VC + LPS $3046 \pm 892.9 \rho \mathrm{g} / \mathrm{ml}$ vs. Lir + LPS $2398 \pm 761.6 \rho \mathrm{g} / \mathrm{ml}, P<0.05)$ (Fig. 1B). Next, gene expression and secretion of the proinflammatory cytokine TNF- $\alpha$ were analyzed in healthy PBMC-derived macrophages. TNF- $\alpha$ mRNA expression was significantly reduced in LPS-stimulated human macrophages treated with Lir compared with VC (VC + LPS 154.4- \pm 36.39-fold change vs. Lir + LPS 106.4- \pm 33.32-fold change, $P<0.05$ ) (Fig. 1C), and TNF- $\alpha$ secretion was significantly decreased in LPS-stimulated PBMC-derived macrophages treated with Lir compared with VC (VC + LPS $804.7 \pm 201.5 \rho \mathrm{g} / \mathrm{ml}$ vs. Lir + LPS $223.0 \pm 72.7 \rho \mathrm{g} / \mathrm{ml}, n=12, P<0.001$ ) (Fig. 1D). In addition, Lir significantly reduced IL-1 $\beta$ secretion after LPS stimulation $(\mathrm{VC}+\mathrm{LPS} 72.9 \pm 17.5 \rho \mathrm{g} / \mathrm{ml} \mathrm{vs}$. Lir + LPS $35.5 \pm 15.3 \rho \mathrm{g} / \mathrm{ml}$, 

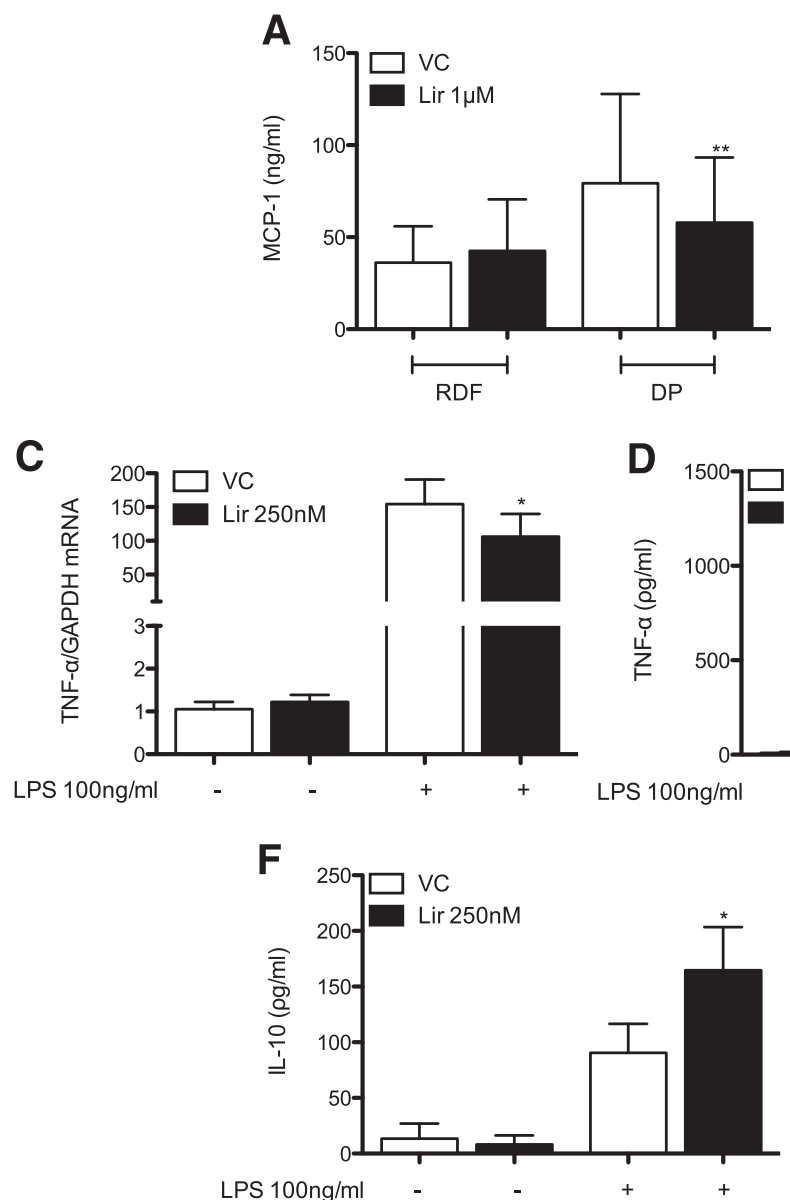
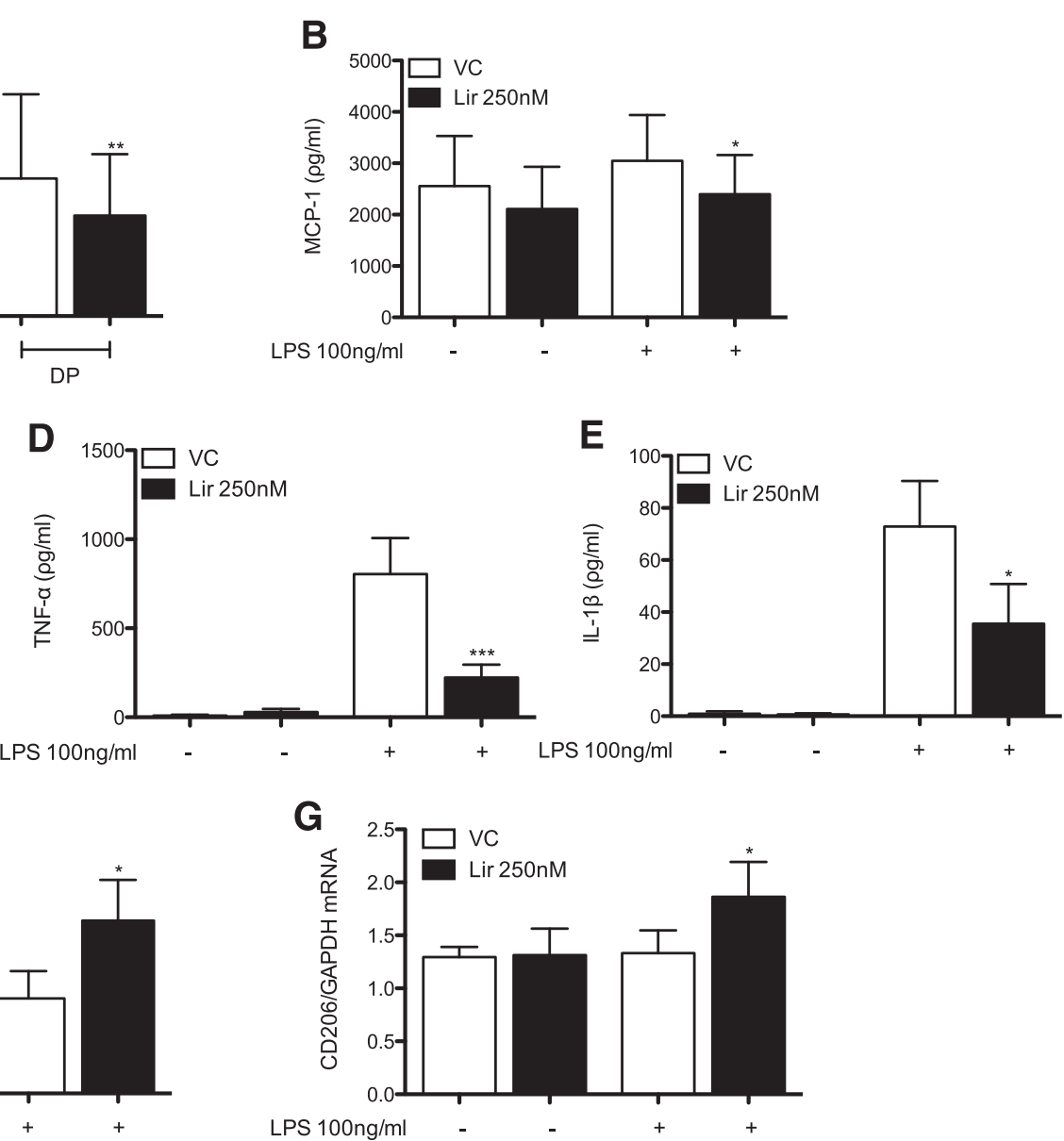

Fig. 1. Proinflammatory mediators are reduced and anti-inflammatory mediators increased in human atherosclerotic plaques and human PBMC-derived macrophages. (A) MCP-1 secretion from RDF and DP sections of carotid ( $n=6)$ and femoral plaques $(n=5)$ were treated with $1 \mu \mathrm{M}$ Lir or VC PBS for 28 hours. Human PBMC-derived macrophages were treated with $250 \mathrm{nM}$ Lir or VC PBS for 6 hours with $100 \mathrm{ng} / \mathrm{ml}$ LPS cotreatment in the final 4 hours and $5 \mathrm{mM}$ ATP cotreatment in the final hour of treatments. We converted mRNA to cDNA, which was analyzed by qRT-PCR using GAPDH as a reference gene, and supernatants were analyzed by ELISA for (B) MCP-1 secretion, (C) TNF- $\alpha$ mRNA, (D) TNF- $\alpha$ secretion, (E) IL-1 $\beta$ secretion, (F) IL-10 secretion, and (G) CD206 mRNA. Error bars represent (A) $n=11,(\mathrm{~B}$ and $\mathrm{C}$ ) and (F and G) $n=7$ and (D and E) $n=12$. Shapiro-Wilk normality tests were carried out to determine whether data were parametric. Paired $t$ tests and Wilcoxon-matched pairs signed rank $t$ tests were used to compare parametric and nonparametric data, respectively, for $\mathrm{VC}$ versus Lir; $* P<0.05$; $* * P<0.01 ; * * P<0.001$ was considered significant; $P>0.05$ was considered nonsignificant.

$n=12, P<0.05)$ in PBMC-derived macrophages (Fig. 1E). Thus, the effect of Lir on inhibiting MCP-1 secretion from human endarterectomy plaques and human macrophages and TNF- $\alpha$ and IL- $1 \beta$ secretion from human macrophages suggests that Lir inhibition of proinflammatory cytokine secretion may alter the atherosclerotic plaque microenvironment to increase a proresolving or anti-inflammatory response. To investigate whether Lir could promote an anti-inflammatory response, IL-10 secretion and CD206 mRNA gene expression were analyzed in human macrophages, where a trend toward increased IL-10 secretion was detected with a significant induction of CD206 mRNA in LPS-stimulated human macrophages treated with Lir (VC + LPS $1.33 \pm 0.21$-fold change, Lir + LPS $1.86 \pm 0.33$-fold change, $P<0.05)($ Fig. $1, F$ and G). To address this issue comprehensively, we used an in vivo model of atherosclerosis regression.

Lir Inhibits Progression of Established Atherosclerosis in $\mathrm{ApoE}^{-/-}$Mice Despite an HFHCD Challenge. After 8 weeks of HFHCD administration to establish extensive aortic lesion burden (Supplemental Fig. 1), $\mathrm{ApoE}^{-1-}$ mice were continued on HFHCDs and treated with either $300 \mu \mathrm{g} / \mathrm{kg}$ of Lir or VC for a further 4 weeks to examine the effect of Lir on established disease. $\mathrm{ApoE}^{-/-}$mice fed LFD for 12 weeks were used as controls. No significant differences in weight, glucose, food intake, or water intake were noted between HFHCD and HFHCD + Lir-treated mice at study completion (Supplemental Fig. 2). Therefore, all data are representative of weight-independent effects, which were anticipated given the dose of Lir used (Gaspari et al., 2013). En face analysis of aortae from the aortic arch to the iliac bifurcation showed HFHCD + Lir-treated $\mathrm{ApoE}^{-1-}$ mice had a marked decrease in whole aorta atherosclerotic lesion burden (HFHCD $6.8 \% \pm 0.3 \%$ vs. HFHCD $+\operatorname{Lir} 4.0 \% \pm 0.4 \%, P<0.001)$ and significant decrease in lesion area in all subsections of the aorta (Fig. 2, A-F) compared with the HFHCD-fed mice. Aortic root lesions were also quantified, and a significant lesion reduction was found in the HFHCD + Lir-treated mice compared with the HFHCD-treated mice (Fig. 2, G and H). Simultaneously, $\mathrm{F} 4 / 80^{+}$macrophage cells were quantified in the aortic root, where a reduction in macrophage content was found in HFHCD + Lir-treated $\mathrm{ApoE}^{-1-}$ mice compared with the HFHCD control, although this result was not significant (Supplemental Fig. 3). This finding clearly demonstrates that 4 weeks of Lir treatment significantly attenuates established disease and may induce resolution of pre-established disease. 
A

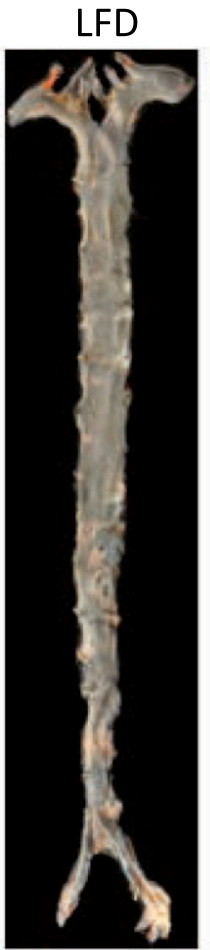

G

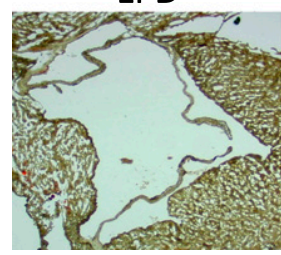

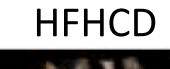

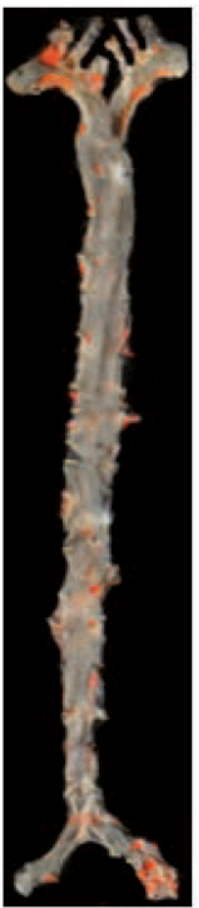

HFHCD+Lir
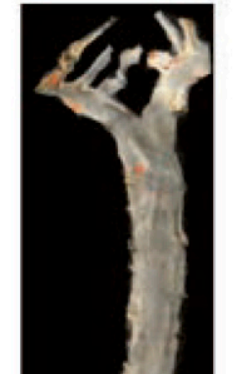

.
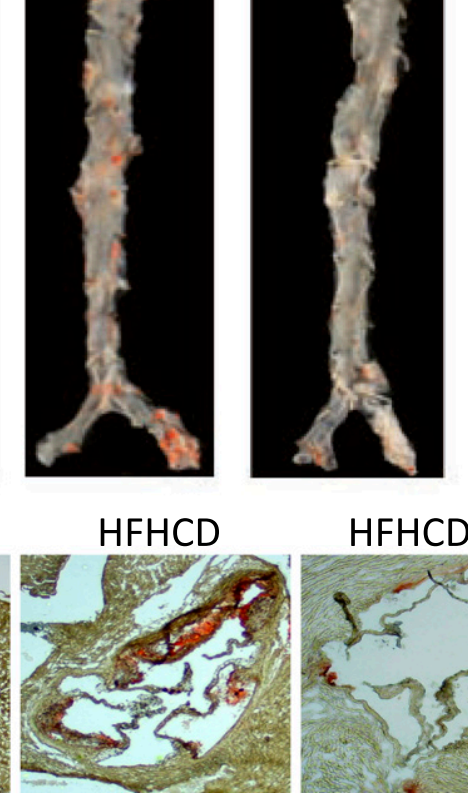

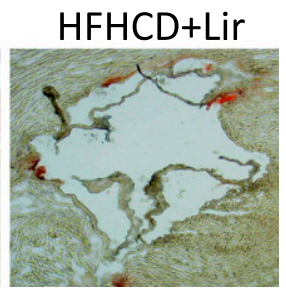

B

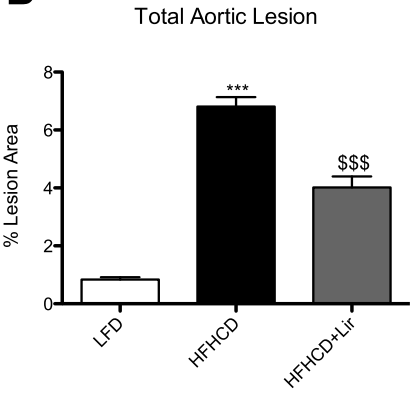

D

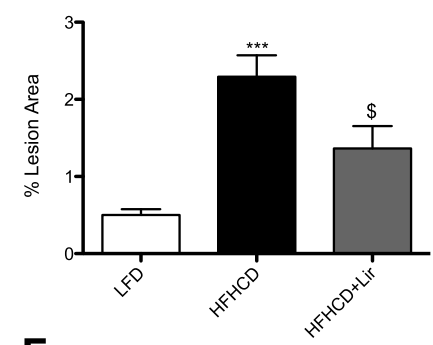

F

Iliac Bifurcation

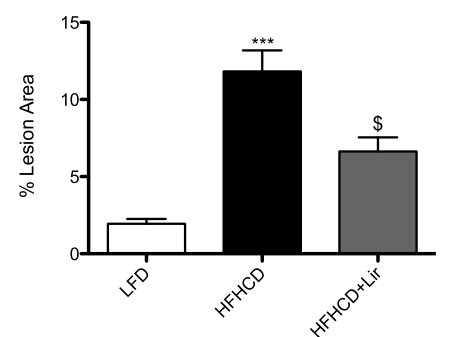

C

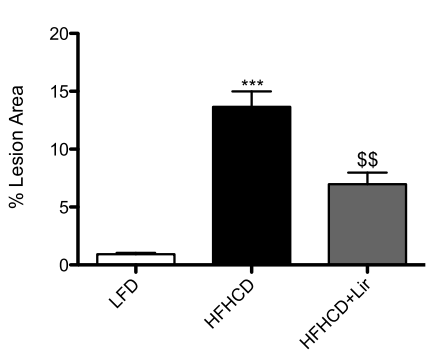

E

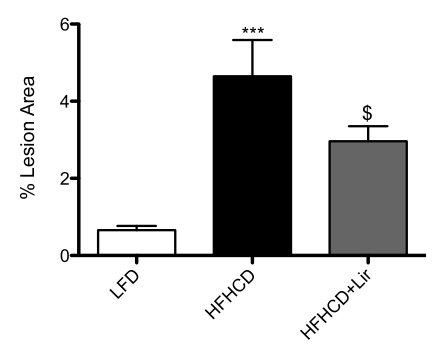

H

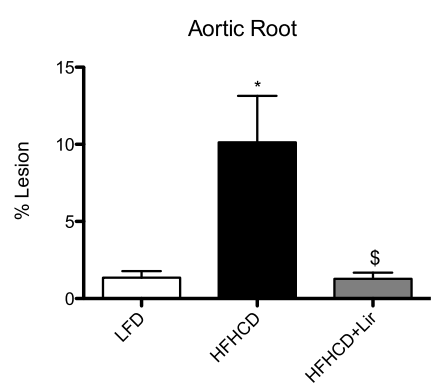

Fig. 2. Lir attenuates pre-established atherosclerosis in vivo in (A) LFD, HFHCD, or HFHCD + Lir-treated ApoE ${ }^{-/-}$aortae stained by en face with Sudan IV for (B) total, (C) aortic arch, (D) thoracic, (E) abdominal aorta, and (F) iliac bifurcation and quantified using ImageJ Software (National Institutes of Health, Bethesda, MD). (G) Aortic roots were sectioned and H| quantified for atherosclerotic lesions using ImageJ. Error bars represent $(\mathrm{A}-\mathrm{F})$ eight mice $(n=8$ per group) and $(\mathrm{G}$ and $\mathrm{H})$ three mice $(n=3)$ per group. Statistical analysis was performed carrying out Mann-Whitney tests between LFD and HFHCD, where statistical significance was considered as follows: $* * * P<0.001 ; * P<0.05$; HFHCD vs. HFHCD + Lir, where ${ }^{\$} P<0.05 ;{ }^{\$} P<0.01 ;{ }^{\$ \$} P<0.001$ was considered significant.

In parallel, aortae were harvested from HFHCD- and HFHCD + Lir-treated $\mathrm{ApoE}^{-1-}$ mice and analyzed for inflammatory cytokine secretion. Although there was a trend toward decreased TNF- $\alpha$ mRNA expression in HFHCD + Lir-treated aortae, Lir significantly decreased aortic $\mathrm{TNF}-\alpha$ secretion (HFHCD $36.50 \pm 14.9 \rho \mathrm{g} / \mathrm{ml}$ vs. HFHCD + Lir $2.21 \pm 2.2 \rho \mathrm{g} / \mathrm{ml}$, $P<0.001$ ) (Fig. 3, A and B). In addition, HFHCD administration induced a significant increase in mRNA expression of the M1 marker iNOS in $\mathrm{ApoE}^{-/-}$aortae, which was blunted in HFHCD + Lir-treated animals (Fig. 3C). Finally, there was a trend toward increased aortic expression of the M2 marker Arg-1, although this trend was not significant (Fig. 3D). Together, these data suggest that Lir may alter the plaque microenvironment to attenuate atherosclerosis.

Proteomic Analysis of Bone Marrow Cells from Lir-Treated ApoE ${ }^{-I-}$ Mice Highlights Enrichment of Proinflammatory Pathways Essential for Regression. To gain insight into the mechanisms through which Lir mediates the atheroprotective effect in attenuating the progression of established atherosclerosis, we performed comprehensive proteomic analysis of bone marrow cells from $\mathrm{ApoE}^{-/-}$ mice, fed LFD for 12 weeks or HFHCD for 12 weeks with either Lir or PBS daily subcutaneous injections for the final 4 weeks of feeding. We found that 561 proteins were significantly altered with HFHCD + Lir (286 upregulated and 275 downregulated) compared with the HFHCD control. Further refinement of the data and elimination of the proteins that were significantly altered between the HFHCD and LFD groups were performed using Perseus version 1.4.1.3 (Supplemental Fig. 4A), resulting in the identification of 338 and 187 significantly altered proteins in the HFHCD and HFHCD + Lir groups, respectively, compared with the LFD control. Importantly, approximately $20 \%$ of the proteins detected were unique to the HFHCD + Lir-treated group (Fig. 4A). Statistical analysis using an unpaired $t$ test identified a unique signature of proteins associated with Lir-mediated inhibition of atherosclerosis (Fig. 4B). The top 25 significantly altered proteins are listed in Supplemental Table 2. Comprehensive bioinformatic analysis using Ingenuity Pathway Analysis was performed on proteins significantly and uniquely regulated by Lir to elucidate potential mechanisms through which Lir mediates its effect. 
A
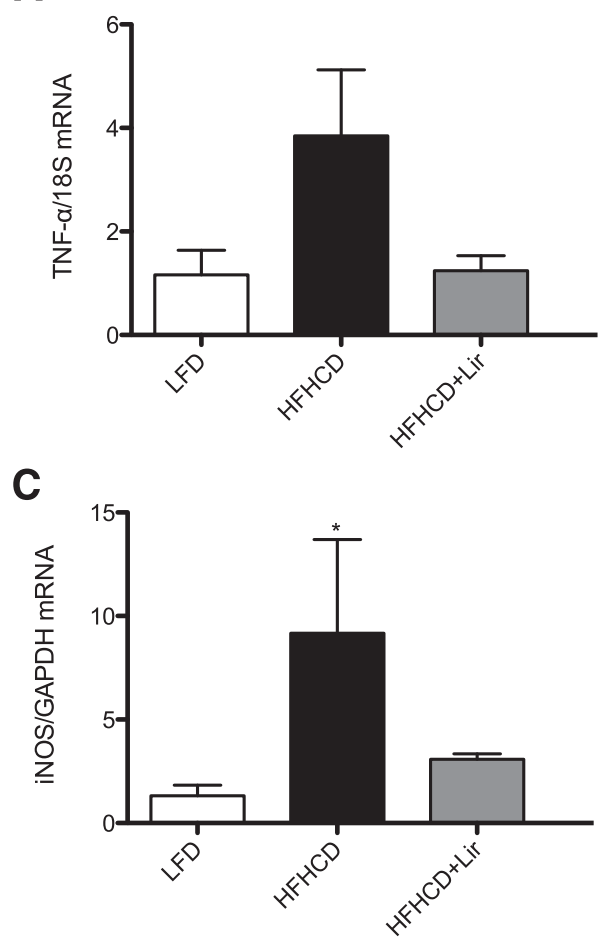

B

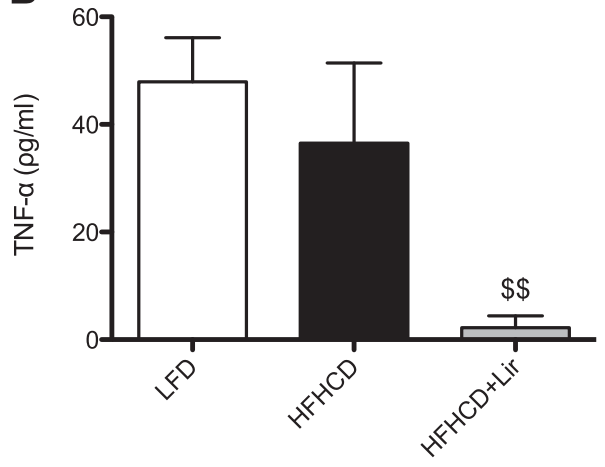

D

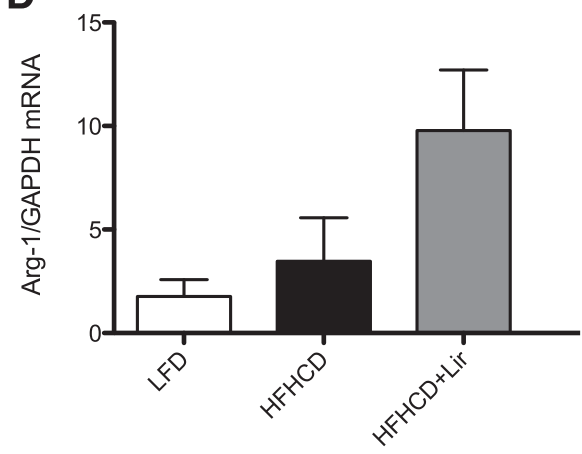

Fig. 3. Lir reduces aortic proinflammatory mediators. ApoE $\mathrm{E}^{-1-}$ aortae were harvested and cultured in RPMI medium, supplemented with $10 \%$ fetal bovine serum and 100 U P-S for 6 hours with aortae and aortic supernatants from LFD-, HFHCD-, and HFHCD + Lir-treated mice analyzed for (A) TNF- $\alpha$ mRNA, (B) TNF- $\alpha$ protein by ELISA, (C) iNOS mRNA, and (D) Arg-1 mRNA. Error bars represent (A and D) $n=6,(\mathrm{~B}) n=7$, and (C) $n=5$. Statistical analysis was performed carrying out Mann-Whitney tests between LFD versus HFHCD in which statistical significance was considered $* P<0.05$ and HFHCD versus HFHCD + Lir; ${ }^{\$ \$} P<0.01$ was considered significant.
Cathepsins are proteolytic macrophage enzymes documented to play a role in protein degradation and atherosclerotic lesion development. For example, CatB degrades the plaque extracellular matrix, and CatZ facilitates proinflammatory cytokine release (Zhao and Herrington, 2016). Surprisingly, proteomic analysis identified CatB and CatZ as significantly enriched proteins in bone marrow cells from HFHCD + Lir-treated mice, despite that these mice having a significantly reduced lesion burden. CatB was identified in a network linked to the insulin receptor and interferon- $\gamma$, both of which are also implicated in atherosclerosis pathogenesis (Fig. 4C). Other proteins significantly upregulated by HFHCD + Lir in this pathway are presented in Supplemental Fig. 4, B-E. Recently, proinflammatory monocytes were shown to be required for regression of atherosclerosis (Rahman et al., 2017), suggesting that enrichment of proinflammatory proteins in monocyte precursor cells may be associated with the inhibition of atherosclerosis observed with Lir-treated animals. Validation of the LFQ proteomic data confirmed a significant increase in bone marrow cell expression of the proatherogenic mediators CatB and CatZ (Fig. 5, A and B).

Previous studies have shown that increased proinflammatory monocytes are required for conversion into M2 proresolving macrophages in the context of atherosclerosis (Rahman et al., 2017). To identify whether this proinflammatory signature was maintained after monocyte-to-macrophage differentiation or macrophages adopted a proresolving phenotype, murine bone marrow cells were differentiated to macrophages. In contrast to what was observed in bone marrow cells, Lir significantly decreased CatB expression (HFHCD $1.0 \pm 0.07$ vs. HFHCD + Lir 0.8- \pm 0.06 -fold change, $P<0.05)$ and did not increase CatZ expression (Fig. 5, C and D) in macrophages. This finding indicates that proinflammatory pathways in bone marrow cells are downregulated when undergoing differentiation to macrophages in the presence of Lir. The mRNA expression of CatB and CatZ were also analyzed in LFD-, HFHCD-, and HFHCD + Lir-treated ApoE ${ }^{-1-}$ aortae. Aortic CatB and CatZ mRNA expression was significantly increased in HFHCD-fed compared with LFD-fed $\mathrm{ApoE}^{-/-}$mice. Interestingly, CatB mRNA expression was significantly reduced, and CatZ mRNA expression was blunted in HFHCD + Lir-treated mice aortae compared with HFHCD-fed animals (Fig. 5, E and F). Thus, it is feasible to hypothesize that Lir mediates its effect by recruiting inflammatory bone marrow cells to differentiate into proresolving macrophages; in accord with recent publications, this effect mediates regression of atherosclerosis (Rahman et al., 2017). Therefore, the next series of experiments were designed to investigate monocyte and macrophage populations in bone marrow and lymphoid tissues during Lirinduced attenuation of established atherosclerosis.

BMDMs from ApoE ${ }^{-/-}$Mice are Atheroprotective with In Vivo Lir Treatment. Apo $\mathrm{E}^{-/-}$bone marrow monocytes and BMDMs were harvested from mice fed a LFD for 12 weeks or a HFHCD for 12 weeks; during the final 4 weeks, mice received daily subcutaneous Lir or VC and were analyzed by flow cytometry for proinflammatory and proresolving phenotypes. Although inflammatory monocytes in HFHCDfed mice decreased significantly compared with LFD controls, monocytes from HFHCD + Lir-treated mice were not significantly different from those in the HFHCD control, and both HFHCD - and HFHCD + Lir-treated mice had significantly more resident monocytes compared with LFD controls (Fig. 6A). The proteomic data also suggest that the inflammatory status of monocytes is increased with Lir, which is in keeping with recent studies, which show inflammatory monocytes convert to proresolving macrophages in atheroprotection (Rahman et al., 2017). Thus, the analysis was extended to investigate the effect of Lir on macrophage populations. As 


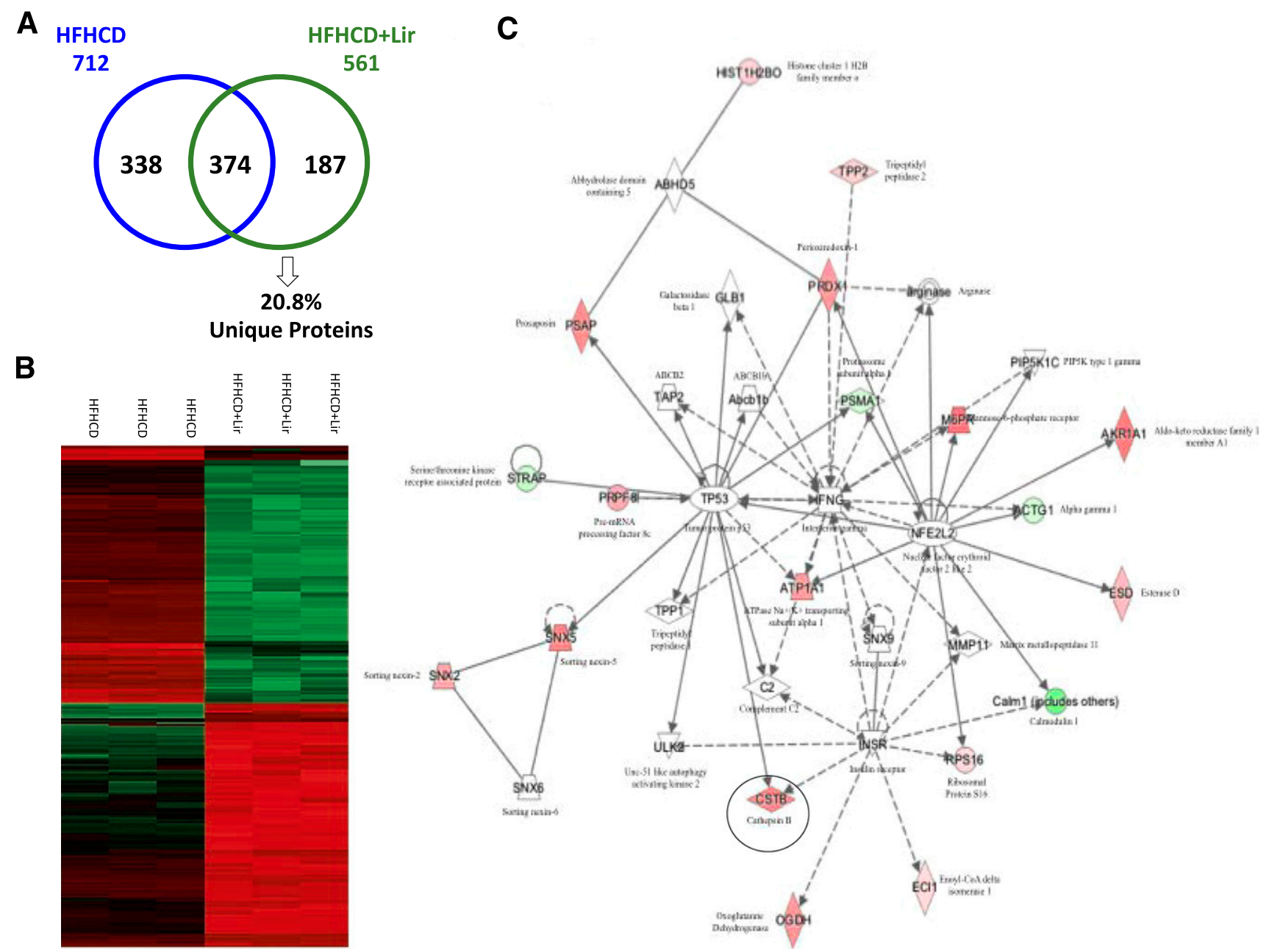

Fig. 4. Proteomic analysis of bone marrow cells during attenuation of pre-established atherosclerosis. ApoE ${ }^{-/-}$bone marrow cells were analyzed by mass spectrometry. (A) Number of proteins was significantly regulated in the treatment groups and were identified using Perseus version 1.4.1.3, (B) proteomic signature heat map of HFHCD vs. HFHCD + Lir bone marrow cells; red and green bars indicate proteins significantly upregulated or downregulated, respectively; $n=3$ per group and (C) identification of regulated network enriched with expression of CatB protein using Ingenuity Pathway Analysis.

expected, the HFHCD-fed mice had significantly increased proinflammatory and decreased anti-inflammatory macrophage populations compared with the LFD control (LFD M1-like $7.3 \% \pm 1.1 \%$ vs. HFHCD M1-like $18.1 \% \pm 3.3 \%$; LFD M2-like $92.7 \% \pm 1.1 \%$ vs. HFHCD M2-like $71.3 \% \pm 13.0 \%$, $P<0.01$ ). Importantly, Lir significantly increased M2-like and decreased M1-like macrophages compared with HFHCD-treated mice (HFHCD M1-like $18.1 \% \pm 3.3 \%$ vs. HFHCD + Lir M1-like $5.8 \% \pm 1.7 \%$; HFHCD M2-like $71.3 \% \pm 13.0 \%$ vs. HFHCD + Lir M2-like $94.2 \% \pm 1.7 \%, P<0.01$ ). Indeed, Lir normalized the M2-like macrophage populations to those comparable with LFD-treated mice (LFD M2-like 92.7\% $\pm 1.1 \%$ vs. HFHCD + Lir M2-like $94.24 \% \pm 1.7 \%$ ) (Fig. 6B). Finally, similar numbers of monocyte:macrophage ratio in the bone marrow from all animal groups (Fig. 6C), suggest that Lir does not influence reduction in monocyte to macrophage number, but it induces significant alterations in M2 macrophage phenotypes.

Lir Alters the Phenotype of Lymphoid Cells. Antiinflammatory macrophages are depleted during atherosclerotic disease progression, with proinflammatory plaque macrophages dominating in the developing lesion (de Gaetano et al., 2016). Further flow cytometry analysis showed that splenic M2 macrophages were depleted after HFHCD administration compared with LFD controls as determined by a significant inhibition of M2 phenotype (LFD M2 $4.3 \times 10^{7} \pm 2.3 \times 10^{7} \mathrm{cells} / \mathrm{mg}$ vs. HFHCD M2 $4.7 \times 10^{6} \pm 2.5 \times 10^{6}$ cells $/ \mathrm{mg}, P<0.05$ ). Interestingly there was no significant reduction in M2 splenic macrophages in HFHCD + Lir-treated mice compared with LFD controls, suggesting that Lir blocks the depletion of M2 macrophages observed in the HFHCD mice (Fig. 6D).

Monocytes and dendritic cells (DCs) are normally recruited into advanced atherosclerotic plaques, and egress of macrophages and DCs to regional and systemic LNs occurs in the regression of atherosclerosis (de Gaetano et al., 2015). Flow cytometry analysis of LNs showed a reduction of immature monocytes and DCs and mature DCs in lymphoid tissue from HFHCD compared with LFD controls. Importantly, Lir rescued mature DCs within LNs as seen by an increase in population numbers (Fig. 6E), suggesting emigration of DCs from the resolving plaque. As there is a trend toward increased DC numbers in the draining $\mathrm{LN}$ with Lir, these data suggest that Lir may promote DC egress from atherosclerotic lesions, which is necessary for the induction of plaque regression. 
A

Cathepsin B
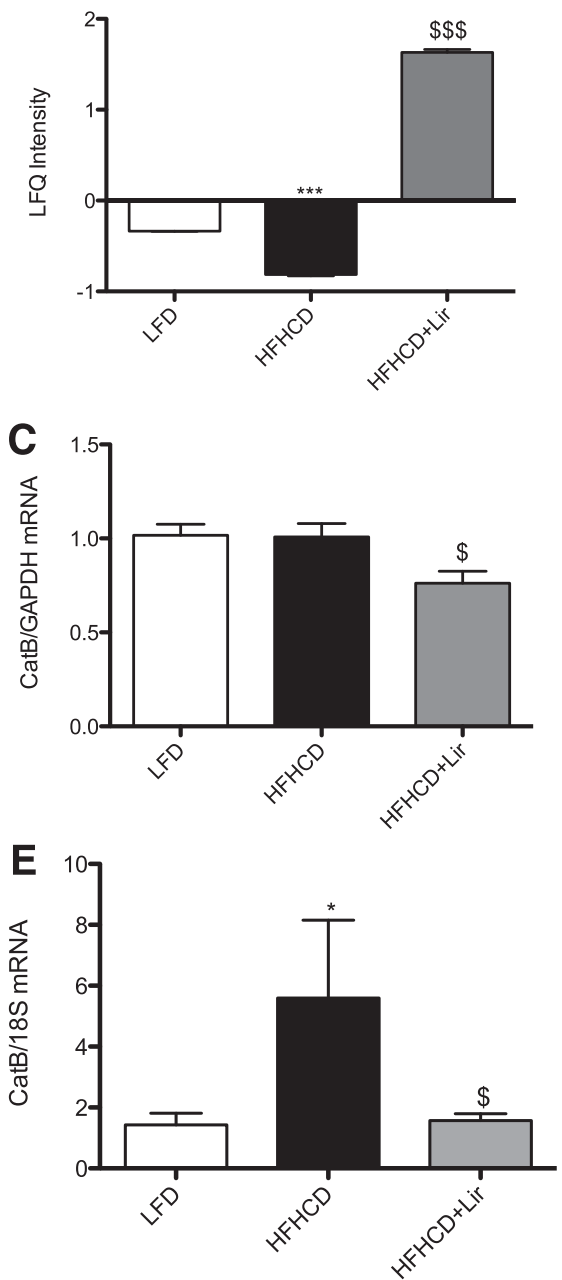

\section{Discussion}

Chronic low-grade inflammation is associated with both diabetes and atherosclerosis; patients with type 2 diabetes mellitus and atherosclerosis have increased proinflammatory monocyte and macrophage populations (Nikiforov et al., 2017). Indeed, chronic inflammatory diseases are associated with a shift in the M1:M2 ratio toward increased proinflammatory M1 (de Gaetano et al., 2016; Ye et al., 2016). This shift in macrophage ratio has also been linked to the development of insulin resistance in adipose tissue (Ye et al., 2016) and in the progression of atherosclerosis from an asymptomatic to symptomatic disease state (de Gaetano et al., 2015).

It was previously thought that inflammatory and resident monocytes give rise to M1 and M2 macrophages, respectively (Nikiforov et al., 2017; Patel et al., 2017). Fadini et al. (2013) have reported that in type 2 diabetes, patients have markedly reduced anti-inflammatory monocytes through dysregulation in bone marrow function, which may have a negative impact on microangiopathy (Fadini et al., 2013); however, recent evidence has identified that inflammatory monocytes are required to induce proresolving macrophages to mediate regression of atherosclerosis (Rahman et al., 2017). The monocyte-derived macrophage cells within atherosclerotic plaques are thought to originate from bone marrow cells as opposed to recruitment of new circulating monocytes (Patel
Fig. 5. Increased Cat protein expression in bone marrow cells is reversed after differentiation to macrophages and in $\mathrm{ApoE}^{-/-}$aortae. $\mathrm{LFQ}$ protein expression of (A) CatB and (B) CatZ from Perseus version 1.4.1.3 analysis. The mRNA expression of (C) BMDM CatB, (D) BMDM CatZ, (E) aortic CatB, and (F) aortic CatZ from LFD, HFHCD, and HFHCD + Lir-treated ApoE ${ }^{-1-}$ mice were analyzed by qRT-PCR using GAPDH and $18 \mathrm{~S}$ rRNA as reference genes. Error bars are representative of $(\mathrm{A}$ and B) $n=3$, (C and D) $n=5$, and (E and F) $n=8$. Statistical analysis was performed carrying out Mann-Whitney tests between LFD vs. HFHCD where statistical significance was considered, with $* P<0.05, * * P<0.01, * * * P<0.001$ and HFHCD vs. HFHCD + Lir, where ${ }^{\$} P<0.05 ;{ }^{\$ \$} P<0.01$ was considered significant. et al., 2017). It has been previously shown that Lir mediates an anti-inflammatory effect in humans as observed by decreased TNF- $\alpha$, IL- $1 \beta$, IL- 6 , and C-reactive protein inhibition (Shiraishi et al., 2012; Hogan et al., 2014). Hence, this may also be of clinical importance in the context of atherosclerosis and diabetes-accelerated atherosclerosis. Recently, the Liraglutide Effect and Action in Diabetes: Evaluation of CV Outcomes Results (LEADER) trial reported that after 3.8 years, the primary outcome of first occurrence of CVD death, nonfatal myocardial infraction, or nonfatal stroke was significantly reduced in Lir-treated patients (Marso et al., 2016).

Our previously published work provided mechanistic insight through which Lir may mediate atheroprotection, where Lir dictated macrophage cell fate toward an M2 pro-resolving macrophage and halted the development of early atherosclerosis in $\mathrm{ApoE}^{-1-}$ mice (Bruen et al., 2017); however, because a significant portion of type 2 diabetes mellitus patients already have established atherosclerotic disease, a therapeutic goal would be to reduce and stabilize disease by blunting progression or even inducing regression of atherosclerosis while maintaining glycemic control. Our hypothesis was that, in established atherosclerosis, Lir would increase the inflammatory status of monocytes, which, when in the plaque milieu would promote a proresolving M2 macrophage that would dominate, reducing proinflammatory responses associated 
A

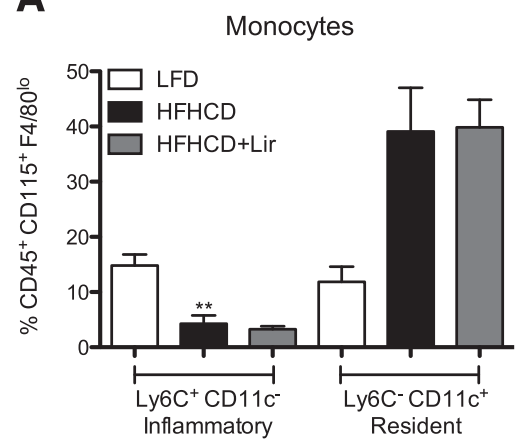

B

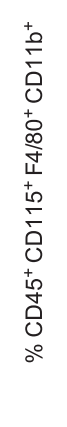

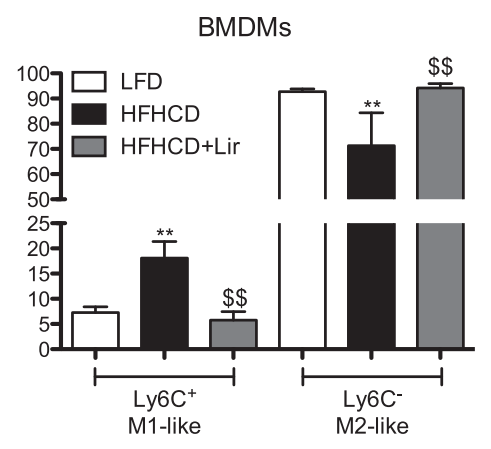

C

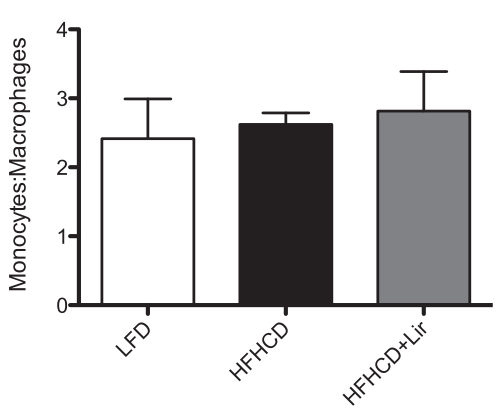

D

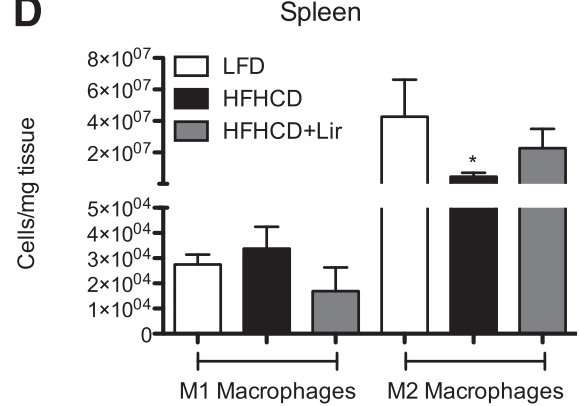

E

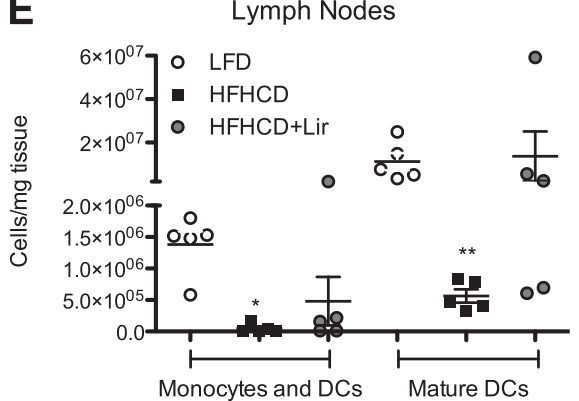

Fig. 6. Lir decreases proatherogenic cell populations in vivo. (A) Bone marrow monocytes, (B) BMDMs, (C) ratio of bone marrow monocytes to BMDMs, (D) splenic macrophages, and (E) LNs were analyzed by flow cytometry. Cells from bone marrow, spleen, and LNs were analyzed according to the antibodies listed in Table 1. Error bars are representative of $(\mathrm{A}-\mathrm{E}), n=5$. Statistical analysis was performed carrying out Mann-Whitney tests between LFD versus HFHCD, where statistical significance was considered, with $* P<0.05$, $* * P<0.01$ and HFHCD versus HFHCD + Lir, where $P<0.01$ was considered significant.

with disease regression and stabilization. The work presented here shows that the inflammatory microenvironment of human atherosclerotic plaques is altered in response to ex vivo Lir treatment, where Lir reduces secretion of MCP-1 from human endarterectomy samples. MCP-1 regulates monocyte chemotaxis and infiltration by binding to the CC chemokine receptor 2 (CCR2) (Ding et al., 2015). Both murine and human studies have shown an association with increased CCR2/MCP-1 expression and atherosclerosis progression (Ding et al., 2015). Indeed, increased circulating MCP-1 levels are related to increased risk of CVD and CVD mortality in patients with coronary artery disease (Ding et al., 2015). As such, strategies for inhibiting MCP-1/CCR2 are currently being explored as a therapeutic goal for the treatment of vascular disorders.

In addition, further analysis on cultured LPS-stimulated inflammatory human PBMC-macrophages showed that Lir significantly decreased secretion of the M1 proinflammatory mediators, MCP-1, TNF- $\alpha$, and IL- $1 \beta$. We also showed Lir could promote anti-inflammatory responses in human macrophages by increasing IL-10 secretion and CD206 gene expression. As Lir altered the inflammatory environment of human atherosclerotic plaques and altered the inflammatory response of macrophage phenotypes in vitro, we hypothesized that Lir may impact the progression of pre-established atherosclerosis. Previous studies investigating GLP-1RAs and their effect on atherosclerosis in mice have been studied in early disease development and progression (Bruen et al., 2017). Here, $\mathrm{ApoE}^{-/-}$mice were fed HFHCD for 8 weeks to establish atherosclerotic disease and maintained on the diet for a further 4 weeks with daily subcutaneous Lir treatment. This is a strength of our study and is more reflective of clinical presentation of established atherosclerosis. Mice were injected with incremental doses of Lir for 10 days to minimize adverse effects and to ensure a dosing regimen similar to that used clinically in human patients. On study completion, aortae were quantified for lesion burden. Lir significantly inhibited lesion burden throughout the aortic root and whole aorta, possibly owing to reduced $\mathrm{F} 4 / 80^{+}$macrophage cells, although this possibility warrants further investigation. Therefore, this is the first report to show that Lir blocks progression of established disease and thus presents us with a unique opportunity to interrogate the potential cellular mechanisms through which Lir mediates its effect on established disease.

Previous studies have shown that lixisenatide alters the plaque milieu by decreasing macrophage content and increasing M2 macrophages in $\mathrm{ApoE}^{-/-}$mice (Vinué et al., 2017), although in low-density lipoprotein receptor knockout mice, Lir did not alter macrophage content or aortic mRNA expression of MCP-1 or iNOS (Bisgaard et al., 2016); however, both studies were performed in the context of early disease progression. Here, we analyzed the plaque microenvironment in established disease and have shown that there is increased TNF- $\alpha$ secretion and aortic iNOS mRNA expression after a 12-week HFHCD challenge. Importantly, Lir administration inhibits aortic TNF- $\alpha$ secretion during attenuation of disease progression.

In atherosclerotic disease progression, monocytes are recruited to the developing lesion and differentiate into macrophages, which sustain the inflammatory response and drive disease progression (Tabas and Lichtman, 2017). As bone marrow cells are recruited into lesions and are precursors for macrophage cells, we characterized these cells in the 
context of attenuated progression of established disease, using proteomic analysis of bone marrow cells from $\mathrm{ApoE}^{-/-}$mice. Bioinformatic analysis showed an upregulation of proinflammatory signaling pathways. This finding is in keeping with recent data showing that inflammatory monocyte precursor cells differentiate into anti-inflammatory macrophages during attenuation of atherosclerosis (Rahman et al., 2017).

Cathepsin proteases are involved in destabilizing atherosclerosis and driving disease progression (Zhao and Herrington, 2016). In bone marrow cells from HFHCD + Lir-treated mice, there was an enrichment of cathepsin proteases despite reduced lesion burden in these mice. Upregulation of this protein family suggests Lir increases the inflammatory status of bone marrow cells, which are precursor cells to monocytes. To address the hypothesis that an increased inflammatory status in bone marrow cells may convert into M2 macrophages, as previously reported in regression studies (Rahman et al., 2017), we next investigated the proinflammatory cathepsin proteins in differentiated macrophages. We show that during differentiation of bone marrow monocytes from $\mathrm{ApoE}^{-/-}$mice treated with HFHCD + Lir, the proinflammatory signature was lost, with no observed increase in CatB or CatZ expression. Indeed, there was a significant decrease in CatB expression in BMDMs from HFHCD + Lir mice. These data correlated with reduced CatB and CatZ mRNA expression in $\mathrm{ApoE}^{-/-}$mice treated with Lir, where aortic CatB expression was significantly decreased. This finding implies that Lir may induce a proinflammatory monocyte, which differentiates into a proresolving macrophage by downregulating proinflammatory pathways in macrophages and the aorta.

Indeed, M2 macrophages have been identified in numerous murine atherosclerosis regression models (Feig et al., 2012). To address whether the loss of the proinflammatory signal in bone marrow cells impacts on macrophage phenotype, we investigated immune cell phenotypes in bone marrow, spleen, and lymph nodes. The data clearly show, as expected, that Lir administration increased proinflammatory proteins in the bone marrow of $\mathrm{ApoE}^{-/-}$mice, suggesting a proinflammatory signature and that this resulted in increased M2-like macrophages upon differentiation. Furthermore, further flow cytometry analysis showed that the proresolving M2 macrophage phenotype prevails both in the bone marrow and in splenic tissue, suggesting that Lir blocks the depletion of M2 macrophages. Finally, we showed a trend toward increased numbers of migratory cells in aortic draining LNs, suggesting increased migratory ability out of lesions, and implies that this may contribute to the ability of Lir to attenuate progression and induce regression of atherosclerosis, although further investigation is warranted.

The data presented here have elucidated an important mechanism in Lir-mediated resolution of inflammation in established atherosclerosis, where proinflammatory bone marrow cells differentiate into proresolving macrophages and likely induce plaque egress of immune cells, promoting repair and inhibiting lesion burden.

\section{Acknowledgments}

We thank the vascular surgery staff and participating patients at St. Vincent's University Hospital (Dublin, Ireland) for providing human carotid plaque material and the UCD Conway Institute Core Facilities, in particular, Catherine Moss, UCD Genomics Core;
Dr. Alfonso Blanco, UCD Flow Cytometry Core; Dr. Dimitri Scholz, UCD Imaging Core; Janet McCormack, UCD Research Pathology Core; and the UCD Proteomics Core for technical support.

\section{Authorship Contributions}

Participated in research design: Bruen, Belton, McGillicuddy. Conducted experiments: Bruen, Curley, Kajani, Lynch, O'Reilly, Dillon.

Contributed new reagents or analytic tools: Barry, Sheehan.

Performed data analysis: Bruen, Dillon, Brennan.

Wrote or contributed to the writing of the manuscript: Bruen, Belton.

\section{References}

Arakawa M, Mita T, Azuma K, Ebato C, Goto H, Nomiyama T, Fujitani Y, Hirose T, Kawamori R, and Watada H (2010) Inhibition of monocyte adhesion to endothelial cells and attenuation of atherosclerotic lesion by a glucagon-like peptide-1 receptor agonist, exendin-4. Diabetes 59:1030-1037.

Bisgaard LS, Bosteen MH, Fink LN, Sørensen CM, Rosendahl A, Mogensen CK, Rasmussen SE, Rolin B, Nielsen LB, and Pedersen TX (2016) Liraglutide reduces both atherosclerosis and kidney inflammation in moderately uremic LDLr-/mice. PLoS One 11:e0168396.

Bornfeldt KE and Tabas I (2011) Insulin resistance, hyperglycemia, and atherosclerosis. Cell Metab 14:575-585.

Brennan EP, Mohan M, McClelland A, Tikellis C, Ziemann M, Kaspi A, Gray SP, Pickering R, Tan SM, Ali-Shah ST, et al. (2018) Lipoxins regulate the early growth response-1 network and reverse diabetic kidney disease. J Am Soc Nephrol 29 $1437-1448$

Bruen R, Curley S, Kajani S, Crean D, O'Reilly ME, Lucitt MB, Godson CG, McGillicuddy FC, and Belton O (2017) Liraglutide dictates macrophage phenotype in apolipoprotein E null mice during early atherosclerosis. Cardiovasc Diabetol 16: 143-156.

de Gaetano M, Alghamdi K, Marcone S, and Belton O (2015) Conjugated linoleic acid induces an atheroprotective macrophage MФ2 phenotype and limits foam cell formation. J Inflamm (Lond) 12:15-29.

de Gaetano M, Crean D, Barry M, and Belton O (2016) M1- and M2-type macrophage responses are predictive of adverse outcomes in human atherosclerosis. Front Immunol 7:275.

Ding D, Su D, Li X, Li Z, Wang Y, Qiu J, Lin P, Zhang Y, Guo P, Xia M, et al. (2015) Serum levels of monocyte chemoattractant protein-1 and all-cause and cardiovascular mortality among patients with coronary artery disease. PLoS One 10: e0120633.

Erbel C, Okuyucu D, Akhavanpoor M, Zhao L, Wangler S, Hakimi M, Doesch A, Dengler TJ, Katus HA, and Gleissner CA (2014) A human ex vivo atherosclerotic plaque model to study lesion biology. $J$ Vis Exp 87:1-6.

Fadini GP, de Kreutzenberg SV, Boscaro E, Albiero M, Cappellari R, Kränkel N, Landmesser U, Toniolo A, Bolego C, Cignarella A, et al. (2013) An unbalanced monocyte polarisation in peripheral blood and bone marrow of patients with type 2 diabetes has an impact on microangiopathy. Diabetologia 56:1856-1866.

Feig JE, Vengrenyuk Y, Reiser V, Wu C, Statnikov A, Aliferis CF, Garabedian MJ, Fisher EA, and Puig O (2012) Regression of atherosclerosis is characterized by broad changes in the plaque macrophage transcriptome. PLoS One 7:e39790.

Fujisaka S, Usui I, Bukhari A, Ikutani M, Oya T, Kanatani Y, Tsuneyama K, Nagai Y, Takatsu K, Urakaze M, et al. (2009) Regulatory mechanisms for adipose tissue M1 and M2 macrophages in diet-induced obese mice. Diabetes 58:2574-2582.

Gaspari T, Liu H, Welungoda I, Hu Y, Widdop RE, Knudsen LB, Simpson RW, and Dear AE (2011) A GLP-1 receptor agonist liraglutide inhibits endothelial cell dysfunction and vascular adhesion molecule expression in an ApoE-/- mouse model. Diab Vasc Dis Res 8:117-124.

Gaspari T, Welungoda I, Widdop RE, Simpson RW, and Dear AE (2013) The GLP-1 receptor agonist liraglutide inhibits progression of vascular disease via effects on atherogenesis, plaque stability and endothelial function in an $\mathrm{ApoE}^{(-/)}$mouse model. Diab Vasc Dis Res 10:353-360.

Gerhard GT and Duell PB (1999) Homocysteine and atherosclerosis. Curr Opin Lipidol 10:417-428.

Hogan AE, Gaoatswe G, Lynch L, Corrigan MA, Woods C, O'Connell J, and O'Shea D (2014) Glucagon-like peptide 1 analogue therapy directly modulates innate immune-mediated inflammation in individuals with type 2 diabetes mellitus. Diabetologia 57:781-784.

Lusis AJ (2000) Atherosclerosis. Nature 407:233-241.

Marso SP, Daniels GH, Brown-Frandsen K, Kristensen P, Mann JF, Nauck MA, Nissen SE, Pocock S, Poulter NR, Ravn LS, et al.; LEADER Steering Committee LEADER Trial Investigators (2016) Liraglutide and cardiovascular outcomes in type 2 diabetes. $N$ Engl $J$ Med 375:311-322.

McCarthy C, Duffy MM, Mooney D, James WG, Griffin MD, Fitzgerald DJ, and Belton O (2013) IL-10 mediates the immunoregulatory response in conjugated linoleic acid-induced regression of atherosclerosis. FASEB $J$ 27:499-510.

Nagashima M, Watanabe T, Terasaki M, Tomoyasu M, Nohtomi K, Kim-Kaneyama J, Miyazaki A, and Hirano T (2011) Native incretins prevent the development of atherosclerotic lesions in apolipoprotein E knockout mice. Diabetologia 54: 2649-2659.

Nikiforov NG, Galstyan KO, Nedosugova LV, Elizova NV, Kolmychkova KI, and Ivanova EA (2017) Proinflammatory monocyte polarization in type 2 diabetes mellitus and coronary heart disease. Vessel Plus 1:192-195.

O'Reilly M, Dillon E, Guo W, Finucane O, McMorrow A, Murphy A, Lyons C, Jones D, Ryan M, Gibney M, et al. (2016) High-density lipoprotein proteomic composition, 
and not efflux capacity, reflects differential modulation of reverse cholesterol transport by saturated and monounsaturated fat diets. Circulation 133:1838-1850. Patel AA, Zhang Y, Fullerton JN, Boelen L, Rongvaux A, Maini AA, Bigley V, Flavell RA, Gilroy DW, Asquith B, et al. (2017) The fate and lifespan of human monocyte subsets in steady state and systemic inflammation. J Exp Med 214:1913-1923.

Rahman K, Vengrenyuk Y, Ramsey SA, Vila NR, Girgis NM, Liu J, Gusarova V, Gromada J, Weinstock A, Moore KJ, et al. (2017) Inflammatory Ly6Chi monocytes and their conversion to M2 macrophages drive atherosclerosis regression. J Clin Invest 127:2904-2915.

Rizzo M, Abate N, Chandalia M, Rizvi AA, Giglio RV, Nikolic D, Marino Gammazza A, Barbagallo I, Isenovic ER, Banach M, et al. (2015) Liraglutide reduces oxidative stress and restores heme oxygenase- 1 and ghrelin levels in patients with type 2 diabetes: a prospective pilot study. J Clin Endocrinol Metab 100:603-606.

Ross R (1993) The pathogenesis of atherosclerosis: a perspective for the $1990 \mathrm{~s}$ Nature 362:801-809.

Shiraishi D, Fujiwara Y, Komohara Y, Mizuta H, and Takeya M (2012) Glucagon-like peptide-1 (GLP-1) induces M2 polarization of human macrophages via STAT3 activation. Biochem Biophys Res Commun 425:304-308.
Tabas I and Lichtman AH (2017) Monocyte-macrophages and T cells in atherosclerosis. Immunity 47:621-634.

Vinué Á, Navarro J, Herrero-Cervera A, García-Cubas M, Andrés-Blasco I, MartínezHervás S, Real JT, Ascaso JF, and González-Navarro H (2017) The GLP-1 analogue lixisenatide decreases atherosclerosis in insulin-resistant mice by modulating macrophage phenotype. Diabetologia 60:1801-1812.

Ye L, Liang S, Guo C, Yu X, Zhao J, Zhang H, and Shang W (2016) Inhibition of M1 macrophage activation in adipose tissue by berberine improves insulin resistance. Life Sci 166:82-91.

Zhao CF and Herrington DM (2016) The function of cathepsins B, D, and X in ath erosclerosis. Am J Cardiovasc Dis 6:163-170.

Address correspondence to: Orina Belton, Diabetes Complications Research Centre, School of Biomolecular and Biomedical Science, UCD Conway Institute, University College Dublin, Belfield, Dublin 4, Ireland. E-mail: orina.belton@ucd.ie 